\title{
TANGENTIAL EQUIVALENCE OF GROUP ACTIONS
}

BY

\author{
SEAWOMIR KWASIK
}

\begin{abstract}
We consider the problem of tangential equivalence of group actions on manifolds. In particular we discuss a conjecture of B. Mazur and its modifications. The negative answer to this conjecture is presented. On the other hand we prove that the "isovariant" version of this conjecture, as well as the modified one, remains true.

As an application some results on the tangential equivalence of $Z_{p}$-actions on homotopy spheres are obtained.
\end{abstract}

1. Introduction. In this paper we consider the problem posed by Mazur in [9]. Namely, let $G$ be a finite group and let $M_{1}, M_{2}$ be two smooth $G$-manifolds. Denote by $R^{k}, k \geqslant 0$, the Euclidean $k$-dimensional space. Suppose we have an equivariant homotopy equivalence $f: M_{1} \rightarrow M_{2}$. Our question is: Does there exist a $k \geqslant 0$ and an equivariant diffeomorphism $\Phi: M_{1} \times R^{k} \rightarrow M_{2} \times R^{k}$ (trivial action of $G$ on $R^{k}$ ) such that the following diagram is commutative up to $G$-homotopy:

$$
\begin{array}{ccc}
M_{1} \times R^{k} & \stackrel{\Phi}{\rightarrow} & M_{2} \times R^{k} \\
\downarrow \pi & & \downarrow \pi \\
M_{1} & \stackrel{f}{\rightarrow} & M_{2}
\end{array}
$$

where $\pi: M_{i} \times R^{k} \rightarrow M_{i}, i=1,2$, is the canonical projection.

Observe that the necessary condition for the existence of such an equivariant diffeomorphism is a stable $G$-equivalence of the tangent $G$-bundle $T M_{1}$ and the induced $G$-bundle $f^{*}\left(T M_{2}\right)$.

Let $\rho: G \rightarrow O(k)$ be an orthogonal representation of $G$. An equivariant homotopy equivalence $f: M_{1} \rightarrow M_{2}$ with the property $T M_{1} \oplus \theta^{k} \approx_{G} f^{*}\left(T M_{2}\right) \oplus \theta^{k}$, where $\theta^{k}$ is a trivial $G$-bundle $M_{1} \times \rho \rightarrow M_{1}$, and $\approx_{G}$ means $G$-isomorphic, is called equivariantly tangential. When the representation $\rho$ is trivial, the $G$-bundle $M_{1} \times \rho \rightarrow M_{1}$ will be called specially trivial and will be denoted by $\varepsilon^{k}$. When $T M_{1} \oplus \varepsilon^{k}$ $\approx_{G} f^{*}\left(T M_{2}\right) \oplus \varepsilon^{k}, f$ will be called specially equivariantly tangential.

Now we can formulate the conjecture posed by Mazur (see [9, pp. 379-380]).

CONJECTURE. Let $G$ be a finite group and $M_{1}, M_{2}$ two closed (= compact, without boundary), connected, $n$-dimensional, smooth $G$-manifolds. Suppose $f: M_{1} \rightarrow M_{2}$ is a special equivariant tangential homotopy equivalence. Then there exist $k \geqslant 0$ and an

Received by the editors November 23, 1982 and, in revised form, March 31, 1983.

1980 Mathematics Subject Classification. Primary 57S15.

$K e y$ words and phrases. $G$-manifold, equivariant vector bundle, tangential equivalence. 
equivariant diffeomorphism $\Phi: M_{1} \times R^{k} \rightarrow M_{2} \times R^{k}$ such that $(*)$ is commutative up to $G$-homotopy.

In this paper we give a negative answer to the conjecture. A counterexample is constructed using an equivariant concordance between high-dimensional knots. On the other hand some positive results are also presented. Namely, we give an affirmative answer to the "isovariant" version of the conjecture and prove the conjecture is true when we assume $f: M_{1} \rightarrow M_{2}$ is an isovariant homotopy equivalence. In fact we are able to prove a little bit more. We prove that the "isovariant" Mazur conjecture remains true in the case of noncompact manifolds. Analogous results are obtained in the equivariant PL category as well.

We also discuss a modification of the conjecture, namely we replace the Euclidean space $R^{k}$ by an orthogonal representation of $G$. In this case we show that such a modified conjecture remains true in full generality (without the isovaraint condition on $f$ ).

As an application of our considerations, some results on the tangential equivalence of homotopy linear $Z_{p}$-actions on homotopy spheres are given.

ACKNOWLEDGEMENT. This work was done while the author was visiting the University of Heidelberg. I am grateful to Professor Dieter Puppe for the opportunity to work there.

2. $G$-spaces. We recall some fundamental properties of $G$-spaces ( $G$ a compact Lie group). A map $f: X \rightarrow Y$ between two $G$-spaces is called equivariant or a $G$-map if $f g(x)=g f(x)$ for every $x \in X, g \in G$. Let $x \in X$. By $G_{x}=\{g \in G \mid g(x)=x\}$ we will denote the isotropy subgroup of $G$ at $x \in X$, and by $G(x)=\{g(x) \in G \mid g \in G\}$ the orbit of $x \in X$. An equivariant map $f: X \rightarrow Y$ between two $G$-spaces is called isovariant if $G_{x}=G_{f(x)}$ for every $x \in X$. Let $X$ be a $G$-space and $H \subseteq G$ a subgroup of $G$. The set of fixed points of $H$ on $X$ is denoted by

$$
X^{H}=\{x \in X \mid h(x)=x \text { for every } h \in H\} .
$$

Definition. Let $X$ be a $G$-space and $x \in X$. The conjugacy class $\left(G_{x}\right)$ of $G_{x}$ is called the type of the orbit $G(x)$.

The set of all conjugacy classes of subgroups of $G$ is partially ordered as follows:

$\left(H_{1}\right) \leqslant\left(H_{2}\right)$ if some conjugate of $H_{2}$ is a subgroup of $H_{1}$. The union $X_{(H)}$ of all orbits of type $H$ is a $G$-invariant subspace of $X$. Observe that if $f: X \rightarrow Y$ is an isovariant map, it induces an equivariant map $f_{(H)}: X_{(H)} \rightarrow Y_{(H)}$ for every $H \subseteq G$. By

$$
X^{(H)}=G\left(X^{H}\right)=\left\{g(x) \in X \mid g \in G \text { and } x \in X^{H}\right\}
$$

we will denote the saturation of $X^{H}$. It is a $G$-invariant and closed subspace of $X$.

The partial order of orbit types on $X$ induces a partial order on the set of $G$-components of $X^{(H)}, H \subseteq G$. Namely, let $\left\{\left(H_{i}\right), i \in I\right\}$ be the orbit types of $X$, and for each $i \in I$ let $\left\{X_{j}^{\left(H_{i}\right)}, j \in I(j)\right\}$ be the $G$-components of $X^{\left(H_{i}\right)}$. Partially order the $\left\{X_{j}^{\left(H_{i}\right)}\right\}$ by inclusion. If $\left(H_{k}\right) \leqslant\left(H_{1}\right)$ then $X^{\left(H_{k}\right)} \subseteq X^{\left(H_{1}\right)}$ and the $G$-components of $X^{\left(H_{h}\right)}$ are contained in the $G$-components of $X^{\left(H_{1}\right)}$. Thus a minimal element of $\left\{X_{j}^{\left(H_{i}\right)}\right\}$ can be written in the form $X_{j}^{\left(H_{i}\right)}$, where $X_{k}^{\left(H_{1}\right)} \leqslant X_{j}^{\left(H_{i}\right)},\left(H_{1}\right) \leqslant$ $\left(H_{i}\right)$, implies $\left(H_{1}\right)=\left(H_{i}\right)$ and $j=k$. It is easy to see that in this case $X_{j}^{\left(H_{i}\right)}$ is a $G$-component of $X_{\left(H_{t}\right)}$ which is a closed subset of $X$. 
Let $\rho: G \rightarrow O(m)$ be an orthogonal representation of $G$. By $S(\rho), D(\rho)$ we will denote, respectively, the sphere and the disc in this representation.

We refer to [2] for more information about $G$-spaces.

3. Equivariant concordance. In this section we give a counterexample to Mazur's conjecture. The main tool in the construction of this counterexample is an equivariant concordance between equivariant high-dimensional knots.

We start with a few words about equivariant knots. $G$ is assumed to be a finite group.

DEFINITION 3.1. An equivariant knot $K=\left(S^{n+2}, \Sigma^{n}\right)$ is a smooth semifree action of $G$ on $S^{n+2}$ with the fixed point set $\Sigma^{n}$ homotopy sphere.

We say that two equivariant knots $K_{0}=\left(S_{0}^{n+2}, \Sigma_{0}^{n}\right), K_{1}=\left(S_{1}^{n+2}, \Sigma_{1}^{n}\right)$ are equivariantly concordant if there exist a semifree action of $G$ on $S^{n+2} \times I$ and an invariant $(n+1)$-dimensional submanifold (fixed point set of the action of $G$ on $\left.S^{n+2} \times I\right) W \subset S^{n+2} \times I$ such that:

(i) $\partial W=\Sigma_{0}^{n} \cup \Sigma_{1}^{n}$;

(ii) the inclusions $\Sigma_{0}^{n} \rightarrow W, \Sigma_{1}^{n} \rightarrow W$ are homotopy equivalences;

(iii) the action of $G$ on $S^{n+2} \times I$ when restricted to $S^{n+2} \times\{0\}$ gives the action of $G$ on $S_{0}^{n+2}$, and when restricted to $S^{n+2} \times\{1\}$ gives the action of $G$ on $S_{1}^{n+2}$.

One can think about the equivariant concordance between $K_{0}$ and $K_{1}$ as a special equivariant $h$-cobordism between $S_{0}^{n+2}$ and $S_{1}^{n+2}$. In fact, the existence of some nontrivial equivariant $h$-cobordism is all that we will need in our construction.

Now let $G=Z_{p}, p \geqslant 2$ a prime number. Let $\rho$ be an $(n+3)$-dimensional orthogonal representation of $Z_{p}$ which leaves the standard $R^{n+1} \subset R^{n+3}$ fixed and is free on the complement $R^{n+3}-R^{n+1}$. The rotation of $R^{n+3}$ around $R^{n+1}$ by a $p$ th root of unit gives such a $\rho$. We will call the pair $K=\left(S^{n+2}(\rho), S^{n}(\rho)\right)$ the standard $Z_{p}$-knot. First we will find some equivariant nontrivial knot $K_{0}=\left(S_{0}^{n+2}, \Sigma_{0}^{n}\right)$ which is equivariantly concordant to the $K=\left(S^{n+2}(\rho), S^{n}(\rho)\right)$. To do this we repeat the construction from [13, p. 348], namely: We attach equivariantly $p$ disjoint 1-handles $h^{(1)}$ to $B^{n+3}(\rho)$ missing $B^{n+1}(\rho)$ (we assume $n \geqslant 2$ ). The group $Z_{p}$ acts semifree on the resulting space $B=B^{n+3}(\rho)+h_{1}^{(1)}+\cdots+h_{p}^{(1)}$ (just by permutation of these handles) with the fixed point set $B^{n+1}(\rho)$. The fundamental group of $B$ is free on generators $\alpha_{1}, \ldots, \alpha_{p}$ corresponding to the handles, and the fundamental group of the complement $B-B^{n+1}(\rho)$ is free with one generator more, the one which links $B^{n+1}(\rho)$. Let us put $\beta$ for this new generator. It is clear that we can find $p$ disjoint simple closed curves $\gamma_{1}, \ldots, \gamma_{p}$ in the boundary of $B$ which miss $B^{n+1}(\rho)$ and are permuted by $Z_{p}$, where each $\gamma_{i}$ represents the word $\gamma_{i}=\alpha_{i}^{2} \beta \alpha_{i}^{-1} \beta^{-1}$. Next we attach equivariantly to $B$ disjoint 2-handles along $\gamma_{i}$ and obtain $B^{n+3}=B+h_{1}^{(2)}$ $+\cdots+h_{p}^{(2)}$. Since $n \geqslant 2, B^{n+3}$ is an $(n+3)$-dimensional disc on which $Z_{p}$ acts semifree with the fixed point set $B^{n+1}(\rho) \subset B^{n+3}$. If we take $K_{0}=\left(\partial B^{n+3}, S^{n}(\rho)\right)$ then we obtain the equivariant knot which is nontrivial (we have

$$
\Pi_{1}\left(\partial B^{n+3}-S^{n}(\rho)\right)=\left(\alpha_{1}, \ldots, \alpha_{p}, \beta ; \alpha_{1}^{2} \beta \alpha_{1}^{-1} \beta^{-1}=\cdots=\alpha_{p}^{2} \beta \alpha_{p}^{-1} \beta^{-1}\right),
$$

which is nontrivial knot group). 
From the construction we see that $K_{0}$ is equivariantly concordant to $K$. In particular we have an equivariant $h$-cobordism between $\partial B^{n+3}$ and $S^{n+2}(\rho)$; this yields the equivariant homotopy equivalence $f: \partial B^{n+3} \rightarrow S^{n+2}(\rho)$. From the existence of the equivariant collar (see [2]) we infer

$$
f^{*}\left(T\left(\partial B^{n+3}\right)\right) \oplus \varepsilon^{1} \approx_{G} T\left(S^{n+2}(\rho)\right) \oplus \varepsilon^{1} ;
$$

hence $f: \partial B^{n+3} \rightarrow S^{n+2}(\rho)$ is specially equivariantly tangential. Now suppose there exists an equivariant diffeomorphism

$$
\phi: \partial B^{n+3} \times R^{k} \rightarrow S^{n+2}(\rho) \times R^{k} .
$$

The existence of such a diffeomorphism implies the existence of the equivariant diffeomorphism

$$
\phi_{(e)}:\left(\partial B^{n+3} \times R^{k}\right)_{(e)} \rightarrow\left(S^{n+2}(\rho) \times R^{k}\right)_{(e)} .
$$

But it is clear that

$$
\left(\partial B^{n+3} \times R^{k}\right)_{(e)}=\left(\partial B^{n+3}-S^{n}(\rho)\right) \times R^{k}
$$

and

$$
\left(S^{n+2}(\rho) \times R^{k}\right)_{(e)}=\left(S^{n+2}(\rho)-S^{n}(\rho)\right) \times R^{k} ;
$$

hence

$$
\begin{aligned}
\Pi_{1}\left(\left(\partial B^{n+3} \times R^{k}\right)_{(e)}\right) & =\Pi_{1}\left(\left(\partial B^{n+3}-S^{n}(\rho)\right)\right) \neq \Pi_{1}\left(\left(S^{n+2}(\rho) \times R^{k}\right)_{(e)}\right) \\
& =\Pi_{1}\left(S^{n+2}(\rho)-S^{n}(\rho)\right)
\end{aligned}
$$

by construction. This contradicts the existence of $\phi$.

REMARK 3.2. Observe that in this construction it was essentially used that the equivariant homotopy equivalence $f: \partial B^{n+3} \rightarrow S^{n+2}(\rho)$ is not isovariant. In fact, in the case of an isovariant homotopy equivalence Mazur's conjecture remains true (compare Theorem 4.1).

4. Equivariant tangential homotopy equivalence. In this section we give the proof of the generalized "isovariant" version of Mazur's conjecture. Our proof relies heavily on Siebenmann's generalization of Mazur's result on tangential homotopy equivalence (see [16]).

THEOREM 4.1. Let $G$ be a finite group and $M_{1}, M_{2}$ connected, n-dimensional, smooth $G$-manifolds (without boundary). Suppose $f: M_{1} \rightarrow M_{2}$ is a smooth map which is a special equivariant tangential isovariant homotopy equivalence. Then there exist $k \geqslant 0$ and an equivariant diffeomorphism $\phi: M_{1} \times R^{k} \rightarrow M_{2} \times R^{k}$ such that the diagram (*) is commutative up to G-homotopy.

Proof. Let $\left\{M_{1, i}^{(H)}\right\}$ be the partially ordered set of $G$-components of $M_{1}^{(H)}$ for $H \subseteq G$. Let $M_{1, i}^{(H)}$ be a minimal element. Then $M_{1, i}^{(H)} / G=\left(M_{1, i}\right)_{(H)} / G$ is a smooth manifold. Since $f: M_{1} \rightarrow M_{2}$ is a special equivariant tangential isovariant homotopy equivalence, there exists a corresponding element $M_{2, i}^{(H)}$ and an ordinary tangential homotopy equivalence $f^{(H)} / G: \quad M_{1, i}^{(H)} / G \rightarrow M_{2, i}^{(H)} / G$ (observe that in this case 
$\left.\operatorname{dim} M_{1, i}^{(H)}=\operatorname{dim} M_{2, i}^{(H)}\right)$. Now, by Theorem 2.3 in [16] we obtain a diffeomorphism

$$
\Phi^{(H)} / G: M_{1, i}^{(H)} / G \times R^{p} \rightarrow M_{2, i}^{(H)} / G \times R^{p}
$$

for $p \geqslant \operatorname{dim} M_{1, i}^{(H)} / G+1$ and the following homotopy commutative diagram:

$$
\begin{array}{ccc}
M_{1, i}^{(H)} / G \times R^{p} & \stackrel{\Phi^{(H)} / G}{\rightarrow} & M_{2, i}^{(H)} / G \times R^{p} \\
\downarrow & & \downarrow \\
M_{1, i}^{(H)} / G & \stackrel{f^{(H)} / G}{\rightarrow} & M_{2, i}^{(H)} / G
\end{array}
$$

The diffeomorphism $\Phi^{(H)} / G$ lifts to the equivariant diffeomorphism

$$
\Phi^{(H)}: M_{1, i}^{(H)} \times R^{p} \rightarrow M_{2, i}^{(H)} \times R^{p}
$$

and we have the diagram

$$
\begin{array}{ccc}
M_{1, i}^{(H)} \times R^{p} & \stackrel{\Phi^{(H)}}{\rightarrow} & M_{2, i}^{(H)} \times R^{p} \\
\downarrow & & \downarrow \\
M_{1, i}^{(H)} & \stackrel{f^{(H)}}{\rightarrow} & M_{2, i}^{(H)}
\end{array}
$$

which is commutative up to $G$-homotopy.

Let $D_{1}, D_{2}$ be the disjoint union of the closed tubular neighborhoods of the minimal elements in $M_{1}$ and corresponding (under $f$ ) elements in $M_{2}$, respectively. We can extend $\Phi^{(H)}$ to the equivariant diffeomorphism $\Phi^{D}, \Phi^{D}: D_{1} \times R^{p} \rightarrow D_{2} \times$ $R^{p}$. In fact, it is possible because of the existence and uniqueness of the equivariant normal bundles (see [2]). Consequently, we obtain the following $G$-homotopy commutative diagram:

$$
\begin{array}{ccc}
D_{1} \times R^{p} & \stackrel{\Phi^{D}}{\rightarrow} & D_{2} \times R^{p} \\
\downarrow & & \downarrow \\
D_{1} & \stackrel{f^{\prime}}{\rightarrow} & D_{2}
\end{array}
$$

In this diagram the map $f^{\prime}$ is the $G$-homotopy equivalence given by the composition $f^{\prime}=l f^{(H)} p$, where $p$ is the bundle projection and $l$ is the inclusion.

Next we will proceed by induction. Let $M_{1, i}^{(H)}$ be such that if $D_{1}$ is the union of the closed tubular neighborhoods of all the elements which are smaller, then $M_{1, i}^{(H)}=$ $\operatorname{cl}\left(\left(M_{1, i}\right)_{(H)}\right)$, where cl $=$ closure, and smaller mean smaller with respect to the partial order we introduced earlier; there exists an equivariant diffeomorphism $\Phi^{D}$ : $D_{1} \times R^{p} \rightarrow D_{2} \times R^{p}$, for some $p \geqslant 0$, and the diagram

$$
\begin{array}{ccc}
D_{1} \times R^{p} & \stackrel{\Phi^{D}}{\rightarrow} & D_{2} \times R^{p} \\
\downarrow & & \downarrow \\
D_{1} & \stackrel{f^{\prime}}{\rightarrow} & D_{2}
\end{array}
$$


which is $G$-homotopy commutative. In general it can happen that the smooth $G$-manifolds $D_{1}, D_{2}$ have corners. But it is not a real problem since we can round these corners equivariantly.

Observe that $\left(M_{1, i}\right)_{(H)}$ is a smooth $G$-manifold meeting $D_{1}$ transversally and

$$
\left(M_{1, i}\right)_{(H)}=K=\operatorname{cl}\left(\left(M_{1, i}\right)_{(H)}-D_{1}\right)
$$

plus a smooth open $G$-collar attached along the common boundary $\bar{\partial} K=\partial K \cap$ $\partial D_{1}$. Analogously $\left(M_{2, i}\right)_{(H)} \times R^{p}$ meets $\Phi^{D}\left(D_{1} \times R^{p}\right)=D_{2} \times R^{p}$ transversally and

$$
\left(M_{2, i}\right)_{(H)} \times R^{p}=L=\operatorname{cl}\left(\left(\left(M_{2, i}\right)_{(H)}-D_{2}\right) \times R^{p}\right)
$$

plus a smooth open $G$-collar attached along the common boundary $\bar{\partial} L=\partial L \cap$ $\partial\left(D_{2} \times R^{p}\right)$. Since the map $f \times$ id: $\left(M_{1, i}\right)_{(H)} \times R^{p} \rightarrow\left(M_{2, i}\right)_{(H)} \times R^{p}$ is a special equivariant tangential homotopy equivalence, it defines the tangential homotopy equivalence $\bar{f}: K / G \times R^{p} \rightarrow L / G \times R^{p}$ by collapsing the collars. The map $\bar{f}$ is homotopic to a map $\overline{\bar{f}}: K / G \times R^{p} \rightarrow L / G \times R^{p}$, which is equal to $\Phi^{D} / G$ on $\bar{\partial} K / G \times R^{p}$. Again by Theorem 2.3 in [16] there exists a diffeomorphism $\Phi / G$ : $K / G \times R^{p} \times R^{t} \rightarrow L / G \times R^{p} \times R^{t}$ for some $t \geqslant 0$ which is equal to $\Phi^{D} / G$ on $\bar{\partial} K / G \times R^{p} \times\{0\}$ (this follows from the proof of Theorem 2.3 in [16]). After lifting of $\Phi / G$ we obtain the equivariant diffeomorphism $\Phi: K \times R^{p+t} \rightarrow L \times R^{p+t}$ and the $G$-homotopy commutative diagram:

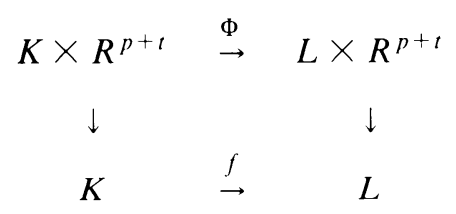

Now we can extend $\Phi$ equivariantly to the closed tubular neighborhoods of $K$ in $M_{1}$ and $L$ in $M_{2}$ as before. This completes the induction step and Theorem 4.1 is proved.

To close this section we give the following example, which is an application of Theorem 4.1.

EXAMPLE 4.2. Let $Z_{p}$ be a cyclic group of order $p$, where $p>2$ is a prime number. Denote by $\rho$ the $n$-dimensional real representation of $Z_{p}$ which is stable and subregular in the sense of [8] and $\operatorname{dim} \rho^{Z_{p}}=k>6$. Put $\Theta_{k}^{\text {fr }}$ for the group of framed homotopy spheres (see $[\mathbf{5}, \mathbf{8}]$ ). Let $F \in \Theta_{k}^{\text {fr }}$ be a homotopy sphere with an exotic smooth structure (such a structure exists because $k>6$; see [5]). By Theorem 6.13 in [8] there exists an action of $Z_{p}$ on the standard sphere $S^{n}$ with the properties:

(i) $\left(S^{n}\right)^{Z_{p}}=F$;

(ii) $T S^{n} \oplus \varepsilon^{1} \approx{ }_{G} S^{n} \times \rho \oplus \varepsilon^{1}$, where $\varepsilon^{1}$ is a specially trivial $Z_{p}$-bundle.

It can be proved (see [14]) that $S^{n}$ with this action is $Z_{p}$-homeomorphic with $S(\rho \oplus 1)$. In fact, this $Z_{p}$-homeomorphism can be taken to be smooth on $S^{n}-p$, where $p \in F=\left(S^{n}\right)^{Z_{p}}$. Observe that in our case $S^{n}$ is not $Z_{p}$-diffeomorphic with $S(\rho \oplus 1)$ because of an exotic smooth structure on $\left(S^{n}\right)^{Z_{p}}$. But it is not difficult to 
see that there exists a smooth isovariant homotopy equivalence $f: S^{n} \rightarrow S(\rho \oplus 1)$. A simple calculation shows that

$$
T S(\rho \oplus 1) \oplus \varepsilon^{1} \approx_{G} S(\rho \oplus 1) \times \rho \oplus 1,
$$

and since $T S^{n} \oplus \varepsilon^{1} \approx_{G} S^{n} \times \rho \oplus \varepsilon^{1}$, we obtain

$$
f^{*}(T S(\rho \oplus 1)) \oplus \varepsilon^{l+1} \approx_{G} T S^{n} \oplus \varepsilon^{l+1} .
$$

Hence by Theorem 3.1 we infer that there exist a $Z_{p}$-diffeomorphism $\Phi: S^{n} \times R^{t} \rightarrow$ $S(\rho \oplus 1) \times R^{t}$ for some $t>0$ and a $Z_{p}$-homotopy commutative diagram:

$$
\begin{array}{ccc}
S^{n} \times R^{t} & \stackrel{\Phi}{\rightarrow} & S(\rho \oplus 1) \times R^{t} \\
\downarrow & & \downarrow \\
S^{n} & \stackrel{f}{\rightarrow} & S(\rho \oplus 1)
\end{array}
$$

5. Equivariant PL manifolds. In this section we consider PL manifolds with PL actions of a finite group $G$. This category lacks certain fundamental properties of the smooth category, but a certain subcategory does have nice properties and this enables us to extend Theorem 4.1 in this context.

Let $G$ be a finite group. All simplicial complexes are assumed to be countable, locally finite and finite dimensional. A $G$-simplicial complex $K$ is a simplicial complex with an action of $G$ on it through simplicial isomorphisms. A $G$ PL space $X$ is a PL space with an action of $G$ on it such that for some triangulation $\psi: K \rightarrow X$ the induced $G$-action on $K$ turns $K$ into a $G$-simplicial complex. Such a $\psi$ will be called a $G$-triangulation of $X$. It is not difficult to observe that the direct generalization of the category of smooth $G$-manifolds by considering only $G$ PL spaces which are PL manifolds is not correct. For example it can be proved (see [1]) that every finite simplicial complex can occur as a fixed point set in a $G$ PL sphere. This (see [14] for a similar discussion) shows some restrictions are needed.

Definition 5.1. A $G$ PL space $X$ is called an $n$-dimensional $G$ PL manifold if for some $G$-triangulation $K$ of $X$ and for every vertex $v$ of $K$, there exist an orthogonal representation $\rho_{v}: G_{v} \rightarrow O(m)$ and a PL $G_{v}$-homeomorphism $\alpha: \operatorname{Lk}(v, K) \rightarrow S\left(\rho_{v}\right)$.

We say that $X$ is an $m$-dimensional $G$ PL manifold with boundary if for every vertex $v$ of $K$ either $\operatorname{Lk}(v, K)$ is PL $G_{v}$-homeomorphic with $S\left(\rho_{v}\right)$ for some $\rho_{v}$ : $G_{v} \rightarrow O(m)$ as above, or there exist an orthogonal representation $\rho_{v}^{\prime}: G_{v} \rightarrow O(m-1)$ and a PL $G_{v}$-homeomorphism $\alpha^{\prime}: \operatorname{Lk}(v, K) \rightarrow D\left(\rho_{v}^{\prime}\right)$.

REMARK. It follows from [3] that every smooth $m$-dimensional $G$-manifold is an $m$-dimensional $G$ PL manifold in the sense of Definition 5.1.

Now let $M_{1}, M_{2}$ be $m$-dimensional $G$ PL manifolds. An equivariant PL map $f$ : $M_{1} \rightarrow M_{2}$ is called equivariantly tangential if $\tau M_{1} \oplus \theta^{k} \approx_{G} f^{*}\left(\tau M_{2}\right) \oplus \theta^{k}$, where $\tau M_{1}, \tau M_{2}$ are tangent $G$ PL microbundles over $M_{1}, M_{2}$, respectively (we refer to [7] for more information about $G$-microbundles). By simple modifications one can 
generalize Theorems 1 and 2 of [15] to the case of $G$ PL manifolds. In particular, we have the following (see [14])

THEOREM 5.2. If $N_{1}, N_{2}$ are two infinite G-regular neighborhoods of the $G \mathrm{PL}$ manifold $X$ in the $G$ PL manifold $M$, there exists an ambient $G$-isotopy of $M$ fixed on $X$ and throwing $N_{1}$ onto $N_{2}$.

When we replace the notion of tubular neighborhood by regular neighborhood we can repeat the proof of Theorem 3.1 in the case of $G$ PL manifolds. Hence, we obtain the following

THEOREM 5.3. Let $M_{1}, M_{2}$ be connected, closed, n-dimensional $G$ PL manifolds. Suppose $f: M_{1} \rightarrow M_{2}$ is a special equivariant tangential, isovariant PL homotopy equivalence. Then there exist $k \geqslant 0$ and an equivariant $\mathrm{PL}$ homeomorphism $\Phi$ : $M_{1} \times R^{k} \rightarrow M_{2} \times R^{k}$ such that the diagram (*) is commutative up to G-homotopy.

6. Modified Mazur conjecture. This section contains the proof of the modified version of Mazur's conjecture (Theorem 6.1 below). The main tool in this proof is the equivariant version of Mazur's (see [9-11]) method of infinite repetition.

TheOREM 6.1. Let $G$ be a compact Lie group and let $M_{1}, M_{2}$ be closed, connected, $n$-dimensional smooth $G$-manifolds. Suppose $f: M_{1} \rightarrow M_{2}$ is an equivariantly tangential homotopy equivalence. Then there exist an orthogonal representation $\rho: G \rightarrow O(m)$ and an equivariant diffeomorphism $\Phi: M_{1} \times \rho \rightarrow M_{2} \times \rho$ such that the diagram analogous to $(*)$ is commutative up to G-homotopy.

Proof. We divide this proof into two parts. First we prove that there exist an orthogonal representation $\rho$ of $G$ and an equivariant embedding $j: M_{1} \rightarrow M_{2} \times \rho$ with the normal $G$-bundle $N(j) G$-isomorphic with the trivial $G$-bundle $M_{1} \times \rho \rightarrow$ $M_{1}$. To see it let $\rho_{1}$ be an orthogonal representation of $G$ for which exist an equivariant embedding $i: M_{1} \rightarrow \rho_{1}$. Take $j_{1}=(f \times i) \Delta: M_{1} \rightarrow M_{1} \times \rho_{1}$, where $\Delta:$ $M_{1} \rightarrow M_{1} \times M_{1}$ is the diagonal map. It is easy to see that $j_{1}$ is an equivariant embedding and $j_{1} \simeq_{G}(f \times$ id $) s$, where $s: M_{1} \rightarrow M_{1} \times \rho_{1}$ is the zero section. Now we compute the normal $G$-bundle $N\left(j_{1}\right)$.

Consider the diagram:

$\begin{array}{ccc}M_{1} \times \rho_{1} & \stackrel{f \times \text { id }}{\rightarrow} & M_{2} \times \rho_{1} \\ s \uparrow \downarrow \pi & \nearrow j_{1} & \downarrow \pi \\ M_{1} & \stackrel{f}{\rightarrow} & M_{2}\end{array}$

Since $j_{1}$ is equivariantly homotopic with $(f \times \mathrm{id}) s$,

$$
j_{1}^{*}\left(T\left(M_{2} \times \rho_{1}\right)\right) \approx_{G} s^{*}(f \times \mathrm{id}) *\left(T\left(M_{2} \times \rho_{1}\right)\right) .
$$

From the equivariant tangentiality of $f$ follows

$$
j_{1}^{*}\left(T\left(M_{2} \times \rho_{1}\right)\right) \oplus \theta^{k} \approx_{G} s^{*}\left(T\left(M_{1} \times \rho_{1}\right)\right) \oplus \theta^{k} \approx_{G} T M_{1} \oplus M_{1} \times \rho_{1} \oplus \theta^{k},
$$


where $\theta^{k}$ is a trivial $G$-bundle $M_{1} \times \rho_{2} \rightarrow M_{1}$ for a $k$-dimensional orthogonal representation $\rho_{2}$ of $G$. On the other hand,

$$
T M_{1} \oplus N\left(j_{1}\right) \oplus \theta^{k} \approx_{G} T M_{1} \oplus M_{1} \times \rho_{1} \oplus \theta^{k} .
$$

If we add to both sides the normal $G$-bundle $N(i)$ of the embedding $i: M_{1} \rightarrow \rho_{1}$, we obtain

$$
T M_{1} \oplus N(i) \oplus N\left(j_{1}\right) \oplus \theta^{k} \approx_{G} T M_{1} \oplus N(i) \oplus M_{1} \times \rho_{1} \oplus \theta^{k},
$$

which we can write in the form

$$
M_{1} \times\left(\rho_{1} \oplus \rho_{2}\right) \oplus N\left(j_{1}\right) \approx_{G} M_{1} \times\left(\rho_{1} \oplus \rho_{1} \oplus \rho_{2}\right) .
$$

Using this we obtain an embedding $j: M_{1} \rightarrow M_{2} \times \rho$, where $\rho=\rho_{1} \oplus \rho_{1} \oplus \rho_{2}$, with the normal $G$-bundle $N(j) G$-isomorphic with $M_{1} \times \rho$.

The second part of the proof of Theorem 6.1 is similar to the proof of the nonequivariant case (see [6]) so we only give its sketch.

Denote by $D_{r}(\rho) \subset \rho$ the standard closed disc with radius $r$. Using the first part of our proof we can obtain two equivariant embeddings,

$$
g_{1}: M_{1} \times D_{r}(\rho) \rightarrow \operatorname{Int} M_{2} \times D_{r}(\rho), \quad g_{2}: M_{2} \times D_{r}(\rho) \rightarrow \text { Int } M_{1} \times D_{r}(\rho),
$$

which are mutually homotopy inverse and such that the equivariant maps $g_{1} s$ : $M_{1} \rightarrow M_{2} \times D_{r}(\rho), j: M_{1} \rightarrow M_{2} \times D_{r}(\rho)$ are equivariantly homotopic. To complete the proof of Theorem 6.1 it suffices to prove

$$
M_{1} \times \text { Int } D_{r}(\rho) \approx_{G \text {-diff }} M_{2} \times \text { Int } D_{r}(\rho) .
$$

To do this we use the equivariant version of the infinite repetition method (it is easy to see this method works, in the equivariant case; see [6]). This, together with the equivariant isotopy theorem (see [2, Theorem 3.1, p. 313]) and the equivariant uniqueness of $G$-tubular neighborhoods (see [2]), gives the proof of Theorem 6.1 (see [6] in the nonequivariant case).

Let us give an application of Theorem 6.1.

EXAMPLE 6.2. Let $M^{n+k}$ be a homotopy sphere with a homotopy linear smooth action of $Z_{p}$, where $p$ is a prime $>2$. Recall that a homotopy linear action means $F^{k}=M^{Z_{p}}$ is a homotopy sphere, too. Suppose $k \geqslant 5, n \geqslant 3$. Let $x \in F$ and let $\rho$ be the tangential representation of $G$ at $x \in F$. There is (see [8]) a $Z_{p}$-homotopy equivalence $f: M \rightarrow S(\rho \oplus 1)$ which is equivariantly tangential. Hence by our Theorem 6.1 there exist an orthogonal representation $\sigma$ of $G$ and an equivariant diffeomorphism $\Phi: M \times \sigma \rightarrow S(\rho \oplus 1) \times \sigma$ such that the following diagram is $Z_{p}$-homotopy commutative:

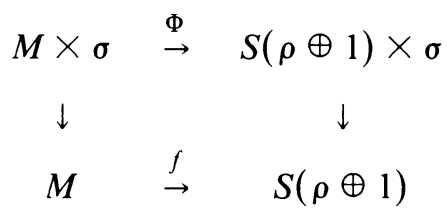

It is really essential that the representation $\sigma$ in the above example is, in general, nontrivial. To see it, let $\rho$ be an $n$-dimensional representation of $Z_{p}$ which is stable in the sense of [8]. Then there exists (see [8]) an action of $Z_{p}$ on the standard sphere $S^{n}$ 
with the nontrivial normal $G$-bundle $\nu\left(\left(S^{n}\right)^{Z_{p}} ; S^{n}\right)$. We know that $S^{n} \times \sigma$ is $Z_{p}$ diffeomorphic with $S(\rho \oplus 1) \times \sigma$ for an orthogonal representation $\sigma$ of $G$. We show that this representation cannot be trivial. In fact, suppose $\sigma=R^{k}$ for some $k>0$ is the trivial representation of $G$ and we have the $Z_{p}$-homotopy commutative diagram

$$
\begin{array}{ccc}
S^{n} \times R^{k} & \stackrel{\Phi}{\rightarrow} & S(\rho \oplus 1) \times R^{k} \\
\downarrow & & \downarrow \\
S^{n} & \stackrel{f}{\rightarrow} & S(\rho \oplus 1)
\end{array}
$$

where $\Phi$ is a $Z_{p}$-diffeomorphism. Then

$$
\begin{aligned}
T S^{n} \oplus \varepsilon^{k} & \approx_{G} f^{*}\left(T(S(\rho \oplus 1)) \oplus \varepsilon^{k}\right) \approx_{G} f^{*}\left(T(S(\rho \oplus 1)) \oplus \varepsilon^{1} \oplus \varepsilon^{k-1}\right) \\
& \approx_{G} f^{*}\left(S(\rho \oplus 1) \times \rho \oplus 1 \oplus \varepsilon^{k-1}\right) \approx_{G} S^{n} \times\left(\rho \oplus 1 \oplus \varepsilon^{k-1}\right) .
\end{aligned}
$$

One can easily prove this implies the $G$-triviality of $\nu\left(\left(S^{n}\right)^{Z_{p}} ; S^{n}\right)$. But this is a contradiction, since we supposed $\nu\left(\left(S^{n}\right)^{Z_{p}} ; S^{n}\right)$ to be nontrivial. Observe that we could assume $S^{n}$ is $Z_{p}$-homeomorphic with $S(\rho \oplus 1)$ (see [14]).

We close this paper with a few remarks.

Remark 6.3. Our proof of the "isovariant" Mazur conjecture does not give any estimation on $k$ in $R^{k}$.

REMARK 6.4. Since an equivariant homotopy equivalence between smooth $G$-manifolds is determined by the ordinary homotopy equivalence between their fixed point sets (see [5]), we can formulate Mazur's conjecture as follows:

Let $G$ be a finite group and $M_{1}, M_{2}$ two closed, connected, $n$-dimensional, smooth $G$-manifolds. Suppose $f: M_{1} \rightarrow M_{2}$ is an equivariant, smooth map such that for every

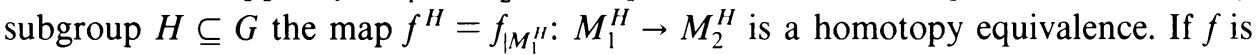
specially equivariantly tangential, there exist $k \geqslant 0$ and an equivariant diffeomorphism $\Phi: M_{1} \times R^{k} \rightarrow M_{2} \times R^{k}$ such that the diagram (*) is $G$-homotopy commutative.

REMARK 6.5. One can ask if this conjecture is true after the full reduction to the nonequivariant homotopy conditions in its formulation. It means: is it enough to require only the ordinary tangentiality for every homotopy equivalence $f^{H}: M_{1}^{H} \rightarrow$ $M_{2}^{H}, H \subseteq G$ ?.

It turns out that such a formulated problem has a negative answer. To see it consider the following example.

EXAMPLE 6.6. Let $L_{1}, L_{2}$ be two 7-dimensional lens spaces obtained by a free action of $Z_{5}$ on the standard 7-dimensional sphere $S^{7}$. Let $L_{1}=L(3 ; 2 ; 2 ; 1)$, $L_{2}=L(2 ; 1 ; 1 ; 1)$ (we have used the notation from [12]). Since $3 \cdot 2 \cdot 2 \cdot 1 \equiv 2 \cdot 1$. $1 \cdot 1(\bmod 5), L_{1}$ and $L_{2}$ are homotopy equivalent (see [12]). Let $f: L_{1} \rightarrow L_{2}$ be a homotopy equivalence. After lifting the map $f$ we obtain the map $\tilde{f}: S_{1}^{7} \rightarrow S_{2}^{7}$ $\left(S_{1}^{7}=S_{2}^{7}=S^{7}\right)$ and the following commutative diagram:

$$
\begin{array}{lll}
S_{1}^{7} & \stackrel{\tilde{f}}{\rightarrow} & S_{2}^{7} \\
\downarrow p & & \downarrow p \\
L_{1} & \stackrel{f}{\rightarrow} & L_{2}
\end{array}
$$


where $p$ is the natural projection. The map $\tilde{f}: S_{1}^{7} \rightarrow S_{2}^{7}$ is the equivariant homotopy equivalence (equivariant with respect to the action of $Z_{5}$ on $\left.S_{1}^{7}, S_{2}^{7}\right)$ and $\tilde{f}^{*}\left(T S_{2}^{7}\right) \approx$ $T S_{1}^{7}$ (since $S^{7}$ is parallelizable).

Now suppose there exist $k \geqslant 0$ and an equivariant diffeomorphism $\Phi: S_{1}^{7} \times R^{k} \rightarrow$ $S_{2}^{7} \times R^{k}$ such that the diagram

$$
\begin{array}{ccc}
S_{1}^{7} \times R^{k} & \stackrel{\Phi}{\rightarrow} & S_{2}^{7} \times R^{k} \\
\downarrow & & \downarrow \\
S_{1}^{7} & \stackrel{\tilde{f}}{\rightarrow} & S_{2}^{7}
\end{array}
$$

is $Z_{5}$-homotopy commutative. In this case we obtain the induced diffeomorphism $\Phi^{\prime}$ : $L_{1} \times R^{k} \rightarrow L_{2} \times R^{k}$ on the orbit spaces and a homotopy commutative diagram:

$$
\begin{array}{ccc}
L_{1} \times R^{k} & \stackrel{\Phi^{\prime}}{\rightarrow} & L_{2} \times R^{k} \\
\downarrow & & \downarrow \\
L_{1} & \stackrel{f}{\rightarrow} & L_{2}
\end{array}
$$

But this cannot be true. In fact, the first Pontryagin class $p_{1}$ of $L_{1}$ is given by $p_{1}\left(L_{1}\right)=23 x^{2}$ and $p_{1}\left(L_{2}\right)=12 x^{2}($ see $[12])$.

\section{REFERENCES}

1. A. Assadi, Finite group actions on simply-connected manifolds and $C W$ complexes, Mem. Amer. Math. Soc. No. 257 (1982).

2. G. Bredon, Introduction to compact transformation groups, Academic Press, New York, 1972.

3. S. Illman, Smooth equivariant triangulations of G-manifolds for $G$ a finite group, Math. Ann. 233 (1978), 199-220.

4. I. M. James and G. B. Segal, On equivariant homotopy type, Topology 17 (1978), 267-272.

5. M. A. Kervaire and J. W. Milnor, Groups of homntopy spheres. I, Ann. of Math. (2) 77 (1963), 504-537.

6. S. Lang, L'equivalence homotopique tangentielle, Séminaire Bourbaki 1960/61, Exp. 217-222.

7. R. Lashof and M. Rothenberg, G-smoothing theory, Proc. Sympos. Pure Math., vol. 32, Part 1, Amer. Math. Soc., Providence, R.I., pp. 211-266.

8. P. Löffler, Homotopielineare $Z_{p}$-operationen auf sphären, Topology 20 (1981), 291-312.

9. B. Mazur, Stable equivalence of differentiable manifolds, Bull. Amer. Math. Soc. 67 (1961), 377-384.

10. , The method of infinite repetition in pure topology. I, Ann. of Math. (2) 80 (1964), 201-226.

11. The method of infinite repetition in pure topology. II, Stable applications, Ann. of Math. (2) 83 (1966), 387-401.

12. J. Milnor, Whitehead torsion, Bull. Amer. Math. Soc. 72 (1966), 358-426.

13. D. Rolfsen, Knots and links, Publish or Perish, Boston, Mass., 1976.

14. M. Rothenberg, Torsion invariants and finite transformation groups, Proc. Sympos. Pure Math., vol. 32, Part 1, Amer. Math. Soc., Providence, R.I., pp. 267-311.

15. A. Scott, Infinite regular neighborhoods, J. London Math. Soc. (2) 42 (1967), 245-253.

16. L. Siebenmann, On detecting open collars, Trans. Amer. Math. Soc. 141 (1969), 201-227.

Mathematisches Institut, Universität Heidelberg, 6900 Heidelberg, Im Neuenheimer Feld 288, WeST GERMANY

InSTitute of Mathematics, University of Gdańsk, 80-952 Gdańsk, Wita Stwosza 57, Poland

Current address: Université de Nantes, Institut de Mathématiques et d'Informatique, 2, Chemin de la Houssiniére, 44072 Nantes-Cedex, France 